Article

\title{
Genome Survey Sequencing of Luffa Cylindrica L. and Microsatellite High Resolution Melting (SSR-HRM) Analysis for Genetic Relationship of Luffa Genotypes
}

\author{
Jianyu An, Mengqi Yin, Qin Zhang, Dongting Gong, Xiaowen Jia, Yajing Guan * and Jin Hu \\ Seed Science Center, Institute of Crop Science, College of Agriculture and Biotechnology, Zhejiang University, \\ 866 Yuhangtang Road, Hangzhou 310058, China; anjianyu@live.cn (J.A.); yinmengi@icloud.com (M.Y.); \\ 21616042@zju.edu.cn (Q.Z.); 21616033@zju.edu.cn (D.G.); 21516035@zju.edu.cn (X.J.); jhu@zju.edu.cn (J.H.) \\ * Correspondence: vcguan@zju.edu.cn; Tel.: +86-0571-8898-2318
}

Received: 26 July 2017; Accepted: 7 September 2017; Published: 11 September 2017

\begin{abstract}
Luffa cylindrica (L.) Roem. is an economically important vegetable crop in China. However, the genomic information on this species is currently unknown. In this study, for the first time, a genome survey of L. cylindrica was carried out using next-generation sequencing (NGS) technology. In total, $43.40 \mathrm{~Gb}$ sequence data of L. cylindrica, about $54.94 \times$ coverage of the estimated genome size of $789.97 \mathrm{Mb}$, were obtained from HiSeq 2500 sequencing, in which the guanine plus cytosine (GC) content was calculated to be $37.90 \%$. The heterozygosity of genome sequences was only $0.24 \%$. In total, 1,913,731 contigs (>200 bp) with 525 bp N $\mathrm{N}_{50}$ length and 1,410,117 scaffolds (>200 bp) with $885.01 \mathrm{Mb}$ total length were obtained. From the initial assembled L. cylindrica genome, 431,234 microsatellites (SSRs) ( $\geq 5$ repeats) were identified. The motif types of SSR repeats included $62.88 \%$ di-nucleotide, $31.03 \%$ tri-nucleotide, $4.59 \%$ tetra-nucleotide, $0.96 \%$ penta-nucleotide and $0.54 \%$ hexa-nucleotide. Eighty genomic SSR markers were developed, and 51/80 primers could be used in both "Zheda 23" and "Zheda 83". Nineteen SSRs were used to investigate the genetic diversity among 32 accessions through SSR-HRM analysis. The unweighted pair group method analysis (UPGMA) dendrogram tree was built by calculating the SSR-HRM raw data. SSR-HRM could be effectively used for genotype relationship analysis of $L$ uffa species.
\end{abstract}

Keywords: genome survey; genomic SSR markers; SSR-HRM; genetic diversity; Luffa (L. cylindrica)

\section{Introduction}

Luffa, or sponge gourd, belonging to the Cucurbitaceae family, is a diploid species with 26 chromosomes $(2 n=26)$ and a cross pollinated crop [1]. Luffa cylindrica (L.) Roem. is one of the most important cultivar species and is mainly planted in tropical and subtropical areas, such as China, Thailand, India, Malaysia, etc. If fruits are harvested at young stage of development, they can be eaten as an edible vegetable, which contains abundant bioactive substances such as alkaloids, flavonoids, sterols, glycosides and glycoprotein to benefit human health [2,3]. When Luffa is fully ripened, the tough fibrous netting from the matured fruit can be used in the bath and kitchen, or as marine steam engine filters and industrial raw materials [4]. Luffa acutangula (L.) Roem. is another species of Luffa genus, which is closely related with Luffa cylindrica (L.) Roem. [1].

Although many studies on Luffa germplasm resources and conventional breeding have been performed, genetic studies are still in their infancy. Currently, no genome survey sequences on Luffa have been reported (as at July 2017), Moreover, only 372 DNA and RNA sequences (https: //www.ncbi.nlm.nih.gov/nuccore/), 41 sequence sets from phylogenetic and population study (https: //www.ncbi.nlm.nih.gov/popset/) and one expressed sequence tag (EST) (https:/ / www.ncbi.nlm.nih. 
gov/nucest/) could be found in National Center for Biotechnology Information (NCBI). The narrow genetic and genomic resources obviously limited the breeding improvement of Luffa.

The recent development of next-generation sequencing (NGS) technologies has produced a large amount of available sequence data. Genome survey sequencing via NGS is an important and cost-effective strategy in generating extensive genetic and genomic information relating to the metabolism and development of organisms [5]. Therefore, to investigate and provide a genomic resource of Luffa for further study, genome survey of L. cylindrica was conducted using NGS technology. These results would be useful for crop improvement programs and better utilization of genomic information in the future [6].

In addition, because of the advantages including decent reproducibility, co-dominance, relative abundance and simplicity, SSR markers have become one of the most useful tools for genetic diversity and linkage mapping analysis. Genomic SSRs and EST SSRs are considered complementary for plant genome mapping [7]. Recently, 1046 pairs of EST-SSR markers were synthesized and verified through transcriptome sequencing in sponge gourd [1,8]. EST-SSRs are useful for genetic analysis; however, their primary limitations are relatively low polymorphism and high possibility of no gene-rich regions in the genome. In contrast, genomic SSRs are highly polymorphic and tend to be widely distributed throughout the genome, resulting in better map coverage [9]. Thus far, no genomic sequence-based markers are available for Luffa.

Meanwhile, high resolution melting (HRM), a sensitive mutation detecting method, has been identified as a powerful, efficient and cost-effective method to analyze genetic variation [10]. It was considered as an evolution of real-time polymerase chain reaction (PCR) technology. During the process of PCR, fluorescent dyes would insert into double-stranded DNA, and accumulate until the end of PCR. Then, with the temperature increasing $0.1^{\circ} \mathrm{C}$ or more per one second from annealing temperature $\left(\sim 60^{\circ} \mathrm{C}\right)$ to relatively high temperature $\left(\sim 95^{\circ} \mathrm{C}\right)$, fluorescence dye separated from DNA, and fluorescence signal was detected at the same time to analyze the Tm values, melting curves and other information. Discrimination of the target amplicon from non-specific products can be done by measuring the difference between Tm values, and the shape of HRM curves [11]. Each HRM curve of amplicon had its own accurate characteristic, which depends on GC content, amplicon length and sequence of the nucleotide sequence [12]. The differences between different amplicons need to be determined after further normalization [13]. Based on the HRM curves, amplicons can be identified even with the same Tm values, and be classified to several categories according to their normalized melting curves and difference plots. Moreover, it allows detection of sequence variants without sequencing or hybridization procedures [14]. The combined analysis HRM and SSR has been used to differentiate highly similar cultivars of sweet cherry [11], lentils (Lens culinaris) [15] and Olea europaea [16]. However, the relevant study on Luffa has not yet been reported.

In this study, L. cylindrica genome sequence produced by genome survey sequencing was reported, which was used to develop a set of new genomic SSR markers of Luffa. The HRM technique efficiency for identifying SSRs in PCR amplifications was assessed and the SSR-HRM method was used to discriminate different Luffa species and cultivars.

\section{Results}

\subsection{Genome Sequencing and Sequence Assembly}

Based on the genome sequence data, $43.40 \mathrm{~Gb}$ clean reads were generated from the small-insert (220 bp) library. The approximate $54.94 \times$ coverage (Table 1) was much better than 30X coverage, which indicated successful assembly [17]. Assembly with K-mer 75 by the SOAPdenovo produced scaffolds with the $\mathrm{N}_{50}$ of $\sim 807 \mathrm{bp}$, and a total length of $\sim 885.01 \mathrm{Mb}$ (Table 2). 
Table 1. Statistics of Luffa cylindrica L. sequencing data.

\begin{tabular}{ccccc}
\hline Library & Data $(G b)$ & Depth $(\times)$ & Q20 (\%) & Q30 (\%) \\
\hline $220 \mathrm{bp}$ & 43.40 & 54.94 & 96.77 & 91.56 \\
\hline
\end{tabular}

Table 2. Statistics of the Luffa cylindrica L. genome assembly.

\begin{tabular}{|c|c|c|c|c|}
\hline Scaffold Number & Scaffold Length (bp) & Scaffold $N_{50}(b p)$ & Scaffold $N_{90}(b p)$ & Gap total Length (bp) \\
\hline $1,410,117$ & $885,010,283$ & 807 & 266 & $9,841,708$ \\
\hline Contig Number & Contig Length (bp) & Contig $\mathrm{N}_{50}(\mathrm{bp})$ & Contig $\mathrm{N}_{90}(\mathrm{bp})$ & GC Content (\%) \\
\hline $1,913,731$ & $875,168,575$ & 525 & 236 & 34.34 \\
\hline
\end{tabular}

The $\mathrm{N}_{50}$ of scaffolds and contigs was calculated by ordering all sequences, then adding the lengths from the longest to shortest until the added length exceeded $50 \%$ of the total length of all sequences. $\mathrm{N}_{90}$ is similarly defined.

\subsection{Genome Size Estimation, GC Content and Genome Survey}

For the 19-mer frequency distribution (Figure 1), the number of K-mers was 37,188,237,568, and the peak of depth distribution was 47.08 . The estimated genome size was $789.97 \mathrm{Mb}$. Similarly, the minor peak at the position of the integer multiples of the main peak indicated a certain repeat rate, and the position at half of the main peak indicated the heterozygosis rate, which were $71.37 \%$ and $0.24 \%$ in L. cylindrica genome, respectively. L. cylindrica had a mid-GC content of $37.90 \%$ (Figure 2).

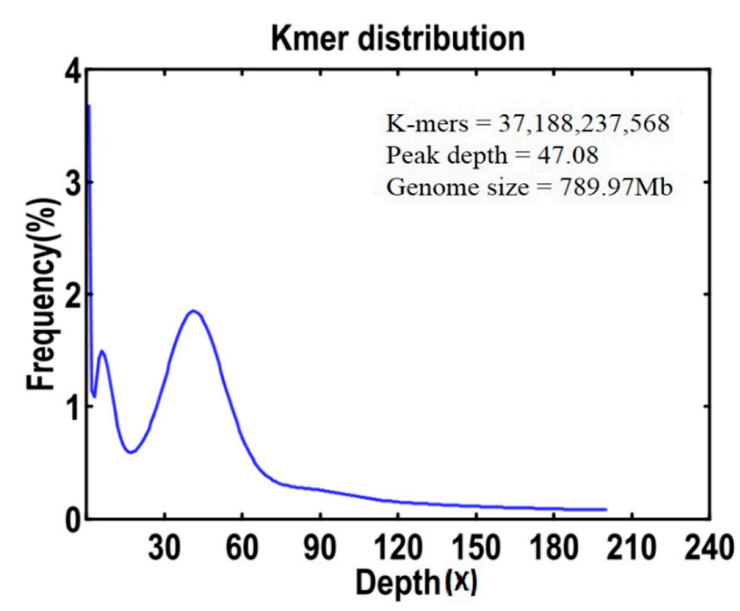

Figure 1. The distribution of $K-$ mer $(K=19)$ analysis based on the whole genome shotgun data in Luffa cylindrica L. (The peak K-mer frequency was 47.08. Genome size was estimated with the formula: estimated genome size $=$ K-mer count $/$ Peak of the depth distribution).



Figure 2. GC content and average sequencing depth of the Luffa cylindrica L. genome data used for assembly. (The $x$-axis was GC content percent across every $10-\mathrm{kb}$ non-overlapping sliding window.) 
From the 1,064,011,890 bp genome survey sequence, 431,234 SSRs were identified (Table 3). The motif length of SSR repeats (without mono-nucleotide) included $62.88 \%$ di-nucleotide, $31.03 \%$ tri-nucleotide, $4.59 \%$ tetra-nucleotide, $0.96 \%$ penta-nucleotide and $0.54 \%$ hexa-nucleotide repeats (Figure 3A). Within the di-nucleotide repeat motifs, the AT/AT accounted for $64.56 \%$, AG/CT for $25.81 \%$, AC/GT for $9.31 \%$ and CG/CG only for $0.32 \%$ (Figure 3B). The predominant tri-nucleotide motifs, AAT/ATT, AAG/CTT and ATC/ATG repeats, respectively, accounted for $55.36 \%, 25.86 \%$ and $5.79 \%$ (Figure $3 \mathrm{C}$ ).

Table 3. Simple sequence repeat types detected in the Luffa cylindrica L. sequences.

\begin{tabular}{ccc}
\hline Searching Item & Number & Percentage \\
\hline Total number of sequences examined & $2,697,125$ & - \\
Total size of examined sequences (bp) & $1,064,011,890$ & - \\
Total number of identified SSRs & 431,234 & $100.00 \%$ \\
Number of SSRs containing sequences & 218,940 & $50.77 \%$ \\
Number of sequences containing more than 1 SSR & 74,313 & $17.23 \%$ \\
Number of SSRs present in compound formation & 47,595 & $11.04 \%$ \\
Mono-nucleotide & 306,140 & $70.99 \%$ \\
Di-nucleotide & 78,655 & $18.24 \%$ \\
Tri-nucleotide & 38,823 & $9.00 \%$ \\
Tetra-nucleotide & 5737 & $1.33 \%$ \\
Penta-nucleotide & 1196 & $0.28 \%$ \\
Hexa-nucleotide & 683 & $0.16 \%$ \\
\hline
\end{tabular}

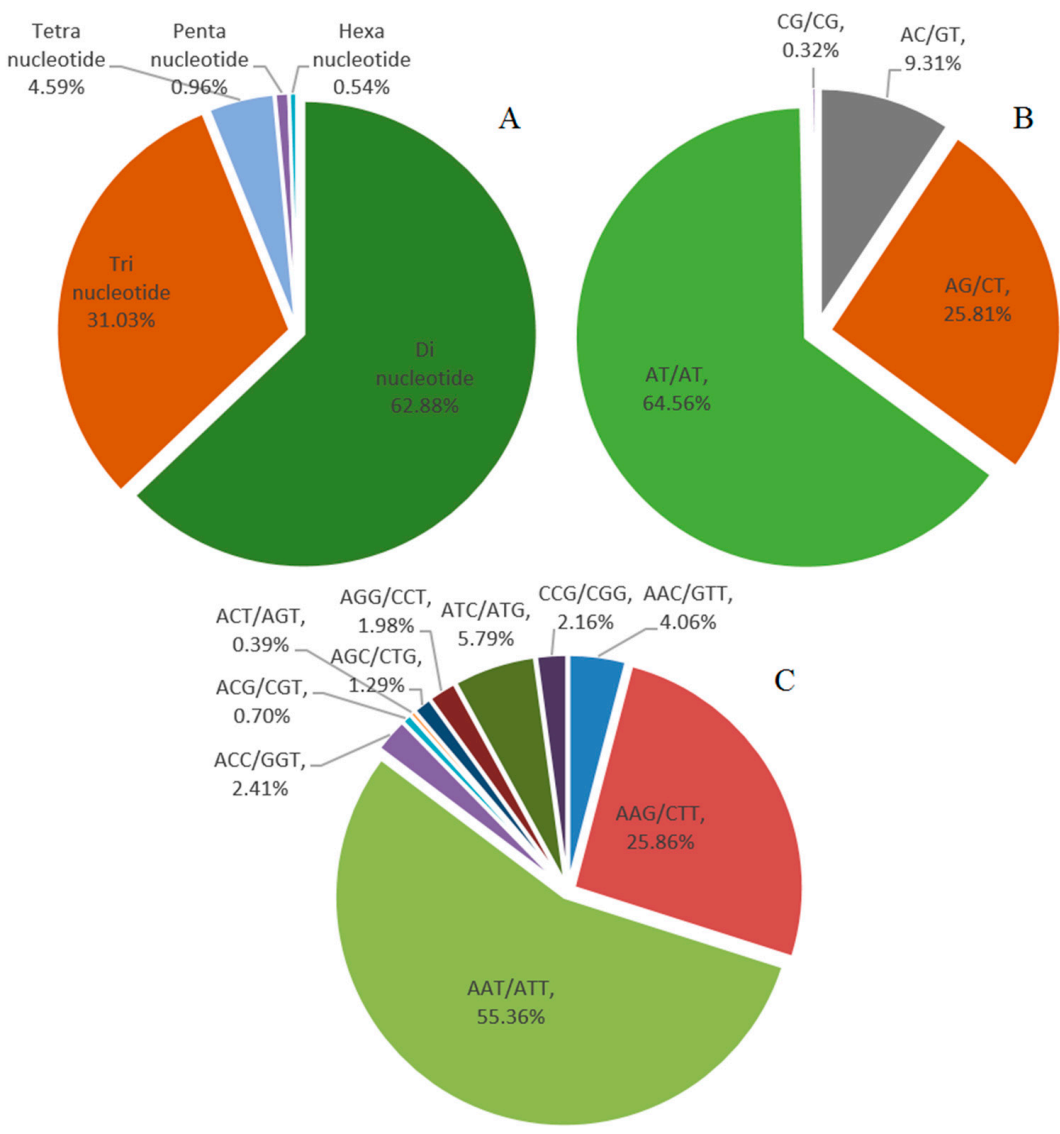

Figure 3. Identification and characteristics of simple repeat sequence (SSR) motifs: (A) frequency of different SSR motif repeat types; (B) frequency of different di-nucleotide SSR motifs; and (C) frequency of different tri-nucleotide SSR motifs. 
SSR motifs categorized by their unit sizes and the number of repeats were summarized (Figure 4). The numbers of di-nucleotide and tri-nucleotide repeats were much more than the other four types. The frequency distribution eliminate range of SSR motif repeats among genomic SSR markers in L. cylindrica ranged 6-37 repeats for di-nucleotide, 5-25 for tri-nucleotide, 5-18 for tetra-nucleotide, 5-15 for penta-nucleotide, and 5-12 for hexa-nucleotide. The variations of repeat numbers decreased with increased motif length.



Figure 4. The distribution and frequency of SSR motif repeat numbers. ( $x$-axis, SSR repeat numbers; $y$-axis, frequency of this SSR type; blue bars, di-nucleotide repeat motifs; yellow bars, tri-nucleotide repeat motif; gray bars, tetra-nucleotide repeat motifs; orange bars, penta-nucleotide repeat motifs; and green bars, hexa-nucleotide repeat motifs.)

\subsection{Genomic SSR Markers Development}

Based on the genome survey of L. cylindrica, 80 genomic SSR primer pairs were designed and synthesized, which were mainly perfect five and six SSR motif repeats. These markers were tested using two inbred cultivars, "Zheda 23" (L. cylindrica) and "Zheda 83" (L. acutangula.). The hybrids between L. cylindrica and L. acutangula had strong heterosis effects [1]. The results in Figure 5 showed that 65 of 80 SSR loci were amplified in "Zheda 23", and 59 of 80 in "Zheda 83", in which, 51 common SSR loci were identified.

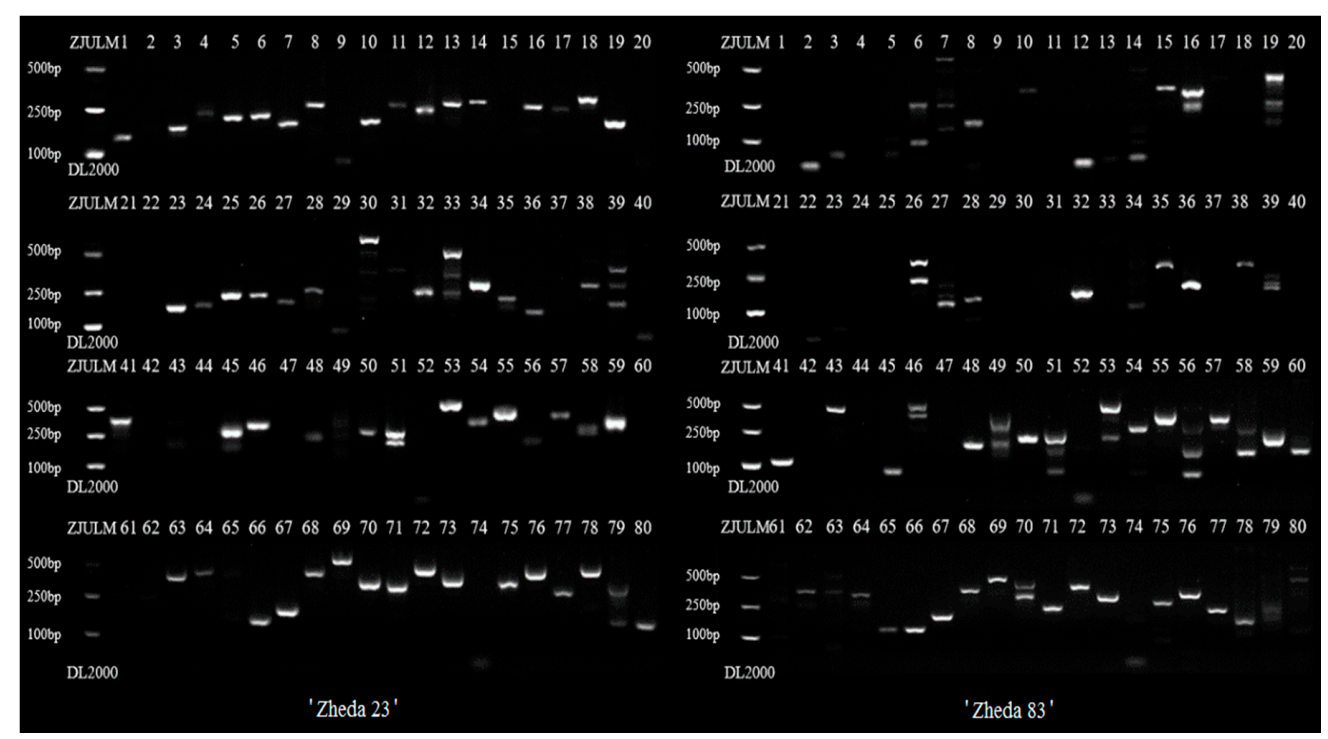

Figure 5. Result of PCR products on 3\% agarose gel at 110V. (Two inbred cultivars, "Zheda 23" (L. cylindrica) and "Zheda 83" (L. acutangula), were tested by 80 Luffa genomic SSR markers). 
Nineteen of fifty-one identified SSR loci were chosen (Table 4) to perform HRM analysis, which were clear enough and could be amplified by both Luffa cultivars. Gene diversity ranged from 0.1913 to 0.8137 , and the PIC at each locus ranged from 0.1730 to 0.7896 with an average of 0.5281 .

Table 4. Characteristics of 19 SSR markers in present study.

\begin{tabular}{|c|c|c|c|c|c|}
\hline Locus & $\begin{array}{l}\text { GenBank } \\
\text { Accession }\end{array}$ & $\begin{array}{l}\text { Repeat } \\
\text { Motif }\end{array}$ & Primer Sequence $\left(5^{\prime}-3^{\prime}\right)$ & $\begin{array}{c}\text { Gene } \\
\text { Diversity }\end{array}$ & PIC \\
\hline ZJULM 3 & KY983987 & (TTTTAT) 6 & $\begin{array}{l}\text { Forward: CGTGTCTTCGGGATAATA } \\
\text { Reverse: CGAAGCATCTTTTACCAT }\end{array}$ & 0.6032 & 0.5556 \\
\hline ZJULM 36 & KY983988 & (CTTCCA)6 & $\begin{array}{l}\text { Forward: CTGAGCAGTCTAACACCCAT } \\
\text { Reverse: TGTGCGAACAAGGAAGGA }\end{array}$ & 0.4141 & 0.3874 \\
\hline ZJULM 39 & KY983989 & (AAAAAT)5 & $\begin{array}{l}\text { Forward: GCGAGTATAGCTCAACGG } \\
\text { Reverse: TTCCAAAATCCAAACCAA }\end{array}$ & 0.3673 & 0.3484 \\
\hline ZJULM 41 & KY983990 & (TGATGG)5 & $\begin{array}{l}\text { Forward: TCGTTGGTGTTGTAGGGTTT } \\
\text { Reverse: GAGGACGAATTGGAAGGAGT }\end{array}$ & 0.6900 & 0.6404 \\
\hline ZJULM 45 & KY983991 & (TTTTA) 5 & $\begin{array}{l}\text { Forward: AATTCCCAGGTAATGTTATG } \\
\text { Reverse: GTCGGCTTGTTTCTTCTC }\end{array}$ & 0.6563 & 0.5888 \\
\hline ZJULM 46 & KY983992 & (AAGAG)5 & $\begin{array}{l}\text { Forward: CCCGCAGTGTTAAGTTTC } \\
\text { Reverse: CCTGCCATGTTTGTTCTC }\end{array}$ & 0.4019 & 0.3756 \\
\hline ZJULM 48 & KY983993 & $(\mathrm{AAAAT}) 5$ & $\begin{array}{l}\text { Forward: TAGAAAGGAAAGGAGGAA } \\
\text { Reverse: TTCAAGAGTTCAGGGTTT }\end{array}$ & 0.6240 & 0.5527 \\
\hline ZJULM 50 & KY983994 & (TAAGA)5 & $\begin{array}{l}\text { Forward: TTCTCCAAATAAGCCACT } \\
\text { Reverse: AGAATCTCCTACCCGTTT }\end{array}$ & 0.8137 & 0.7896 \\
\hline ZJULM 51 & KY983995 & (TGGTTG)6 & $\begin{array}{l}\text { Forward: CCAGTCCAGGAGAAAGGG } \\
\text { Reverse: GAGGCACAACCACAACCA }\end{array}$ & 0.6505 & 0.6129 \\
\hline ZJULM 53 & KY983996 & $($ GAAAGT)5 & $\begin{array}{l}\text { Forward: GCGAAGAGGAGCGAAGAA } \\
\text { Reverse: ATTGGCAATGCAATGAGG }\end{array}$ & 0.3889 & 0.3613 \\
\hline ZJULM 55 & KY983997 & (CCAGCA)5 & $\begin{array}{l}\text { Forward: GATAATGGAAATAAACCACCCT } \\
\text { Reverse: GCCACAGACCCTACTTGAGA }\end{array}$ & 0.7813 & 0.7506 \\
\hline ZJULM 59 & KY983998 & (CСАCСТ)7 & $\begin{array}{l}\text { Forward: CGTGTAGGCTAGGGTCAC } \\
\text { Reverse: CTCCACCACTTCATTTGTAT }\end{array}$ & 0.5898 & 0.5418 \\
\hline ZJULM 66 & KY983999 & (TTTTGT) 5 & $\begin{array}{l}\text { Forward: AAGATCGGTTTGGGAGGA } \\
\text { Reverse: TGGCAGTTTCAGGCAGTC }\end{array}$ & 0.6468 & 0.6156 \\
\hline ZJULM 67 & KY984000 & (GTTTTT)5 & $\begin{array}{l}\text { Forward: GGGATATTGCGGTGGAGT } \\
\text { Reverse: GGTTAGGTGGCGTTCGTC }\end{array}$ & 0.7111 & 0.6637 \\
\hline ZJULM 71 & KY984001 & (ATTTTT) 5 & $\begin{array}{l}\text { Forward: AGTTCCCTGAGCAGATAC } \\
\text { Reverse: CTAAATCAACAACATCCCT }\end{array}$ & 0.5729 & 0.5439 \\
\hline ZJULM 73 & KY984002 & (TGGTTG)6 & $\begin{array}{l}\text { Forward: TTGAGCCTGAGGGATAGA } \\
\text { Reverse: GGATGCTGCTGATAAGTG }\end{array}$ & 0.7041 & 0.6798 \\
\hline ZJULM 77 & KY984003 & (AGAGAA)5 & $\begin{array}{l}\text { Forward: TTCGGTCATTTGATTTCG } \\
\text { Reverse: TTCGTGGAAGAACCCTCT }\end{array}$ & 0.1913 & 0.1730 \\
\hline ZJULM 78 & KY984004 & (AGTTCC)5 & $\begin{array}{l}\text { Forward: GAACATCCCAGGAAATGC } \\
\text { Reverse: GCCAGACGAGGAAGAACA }\end{array}$ & 0.2246 & 0.2096 \\
\hline ZJULM 79 & KY984005 & (GAAAAA)5 & $\begin{array}{l}\text { Forward: GAGGAGATGGTGAGGGAG } \\
\text { Reverse: AACGGATTGCTGATGTGA }\end{array}$ & 0.6806 & 0.6427 \\
\hline Mean & - & - & - & 0.5638 & 0.5281 \\
\hline
\end{tabular}

Shown for each primer pair are the repeat motif, primer sequences, gene diversity, and polymorphism information content (PIC).

\subsection{Genetic Relationship Analysis by SSR-HRM}

For genetic relationship analysis using SSR-HRM method, the genotype of each DNA sample was determined based on the shape of curves depicted by temperature-shifted melting curves and difference plots. In the analysis option of software, both deltaTm discrimination and curve shape sensitivity were set up to $50 \%$. Afterwards, the curves were analyzed, melting curves and difference plots were obtained, and these curves were clustered to several genotype groups. The corresponding accessions would be recorded with their genotype group number (Table 5).

For example, using the genomic SSR marker, Zhejiang University Luffa marker (ZJULM) 50, the difference plots of 32 accessions are shown in Figure 6A and normalized melting curves in Figure 6B. All these cultivars were obviously gathered into eight unique Luffa genotypes. The representative HRM genotype in each group is shown in Figure $6 \mathrm{C}, \mathrm{D}$, which could be easily distinguished visually by their difference plots and melting curves, such as "Zheda 2" (group 3) and "Sanbier" (group 7). Then, each accession was marked with its own genotype group number (Figure $6 \mathrm{E}$ ) for further study. 
The 18 other SSR markers were performed similar to ZJULM 50, and finally the result of these 19 groups of SSR-HRM are presented in Table 5.

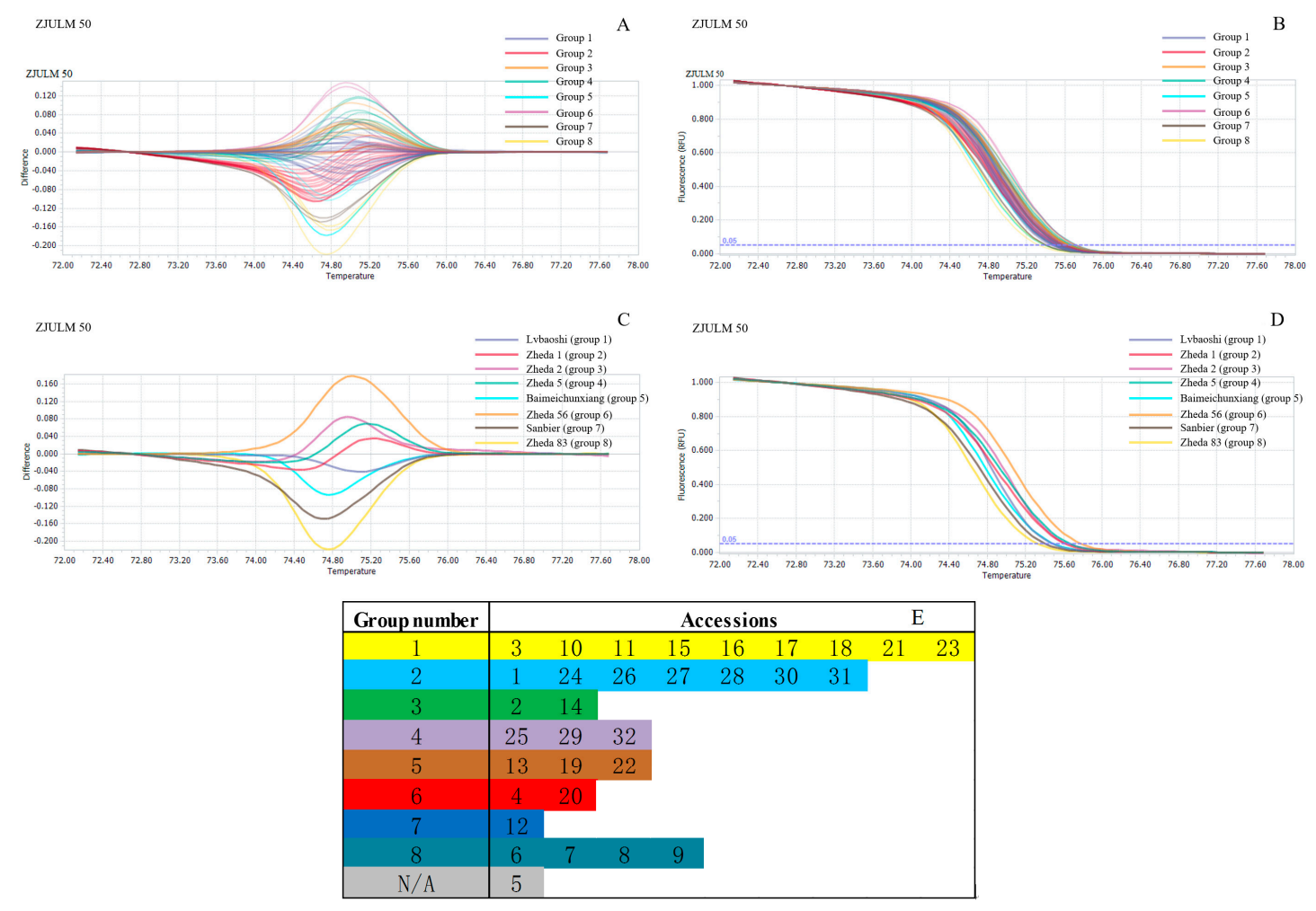

Figure 6. Difference plots and normalized HRM melting curve analyses obtained with the SSR marker ZJULM 50: (A) difference plots of 32 Luffa accessions (each amplicon with three repeats) by the genomic SSR marker ZJULM 50; (B) normalized HRM melting curve of 32 Luffa accessions (each amplicon with three repeats) by the genomic SSR marker ZJULM 50, where the same line color indicates the same genotype calculated by HRM analysis; (C) difference plots of the eight distinguished Luffa accessions by the genomic SSR marker ZJULM 50; (D) normalized HRM melting curve of the eight distinguished Luffa accessions by the genomic SSR marker ZJULM 50; and (E) genotype group number of each accession. Blue dotted line 0.05 in (B) and (D) meant Fluorescence (RFU) $=0.05$.

Table 5. Result of genotype group number obtained with 19 SSR markers.

\begin{tabular}{ccccccccccccccccccccc}
\hline \multirow{2}{*}{$\begin{array}{c}\text { Accession } \\
\text { Number }\end{array}$} & $\mathbf{3}$ & $\mathbf{3 1}$ & $\mathbf{3 9}$ & $\mathbf{4 1}$ & $\mathbf{4 5}$ & $\mathbf{4 6}$ & $\mathbf{4 8}$ & $\mathbf{5 0}$ & $\mathbf{5 1}$ & $\mathbf{5 3}$ & $\mathbf{5 5}$ & $\mathbf{5 9}$ & $\mathbf{6 6}$ & $\mathbf{6 7}$ & $\mathbf{7 1}$ & $\mathbf{7 3}$ & $\mathbf{7 7}$ & $\mathbf{7 8}$ & $\mathbf{7 9}$ \\
\hline 1 & 1 & 3 & 0 & 2 & 2 & 1 & 2 & 2 & 4 & 3 & 6 & 2 & 3 & 3 & 1 & 0 & 1 & 1 & 1 \\
2 & 1 & 1 & 1 & 2 & 2 & 1 & 2 & 3 & 0 & 3 & 1 & 2 & 1 & 1 & 1 & 2 & 1 & 1 & 1 \\
3 & 3 & 1 & 1 & 2 & 2 & 1 & 2 & 1 & 1 & 1 & 5 & 2 & 1 & 1 & 1 & 2 & 1 & 1 & 1 \\
4 & 4 & 1 & 1 & 2 & 2 & 1 & 2 & 6 & 0 & 1 & 5 & 1 & 1 & 1 & 1 & 2 & 1 & 1 & 1 \\
5 & 1 & 1 & 1 & 1 & 2 & 1 & 1 & 0 & 1 & 1 & 6 & 1 & 3 & 1 & 1 & 5 & 1 & 1 & 1 \\
6 & 5 & 2 & 2 & 3 & 1 & 4 & 2 & 8 & 3 & 1 & 2 & 3 & 5 & 4 & 2 & 7 & 1 & 2 & 4 \\
7 & 0 & 2 & 2 & 3 & 3 & 3 & 2 & 8 & 3 & 1 & 2 & 3 & 4 & 4 & 2 & 0 & 1 & 3 & 4 \\
8 & 3 & 2 & 4 & 3 & 0 & 4 & 2 & 8 & 5 & 2 & 2 & 3 & 4 & 5 & 2 & 8 & 1 & 2 & 5 \\
9 & 0 & 2 & 2 & 3 & 4 & 4 & 2 & 8 & 5 & 4 & 2 & 3 & 5 & 4 & 2 & 1 & 1 & 2 & 5 \\
10 & 0 & 1 & 0 & 1 & 1 & 1 & 2 & 1 & 1 & 1 & 1 & 2 & 3 & 3 & 1 & 6 & 1 & 1 & 3 \\
11 & 0 & 1 & 1 & 1 & 1 & 1 & 1 & 1 & 1 & 1 & 4 & 2 & 1 & 3 & 1 & 6 & 1 & 1 & 3 \\
12 & 0 & 3 & 1 & 1 & 2 & 1 & 1 & 7 & 1 & 1 & 1 & 2 & 6 & 3 & 1 & 1 & 1 & 1 & 3 \\
13 & 1 & 1 & 1 & 1 & 1 & 1 & 1 & 5 & 1 & 1 & 5 & 1 & 1 & 3 & 1 & 1 & 1 & 1 & 2 \\
14 & 0 & 1 & 1 & 0 & 2 & 0 & 1 & 3 & 2 & 1 & 4 & 1 & 1 & 2 & 1 & 1 & 1 & 1 & 2 \\
15 & 0 & 1 & 1 & 1 & 1 & 2 & 1 & 1 & 1 & 1 & 4 & 4 & 1 & 1 & 1 & 1 & 1 & 1 & 2 \\
16 & 0 & 1 & 1 & 1 & 1 & 2 & 1 & 1 & 1 & 1 & 1 & 1 & 1 & 1 & 1 & 1 & 1 & 1 & 3 \\
\hline
\end{tabular}


Table 5. Cont.

\begin{tabular}{ccccccccccccccccccccc}
\hline \multirow{2}{*}{$\begin{array}{c}\text { Accession } \\
\text { Number }\end{array}$} & $\mathbf{3}$ & $\mathbf{3 1}$ & $\mathbf{3 9}$ & $\mathbf{4 1}$ & $\mathbf{4 5}$ & $\mathbf{4 6}$ & $\mathbf{4 8}$ & $\mathbf{5 0}$ & $\mathbf{5 1}$ & $\mathbf{5 3}$ & $\mathbf{5 5}$ & $\mathbf{5 9}$ & $\mathbf{6 6}$ & $\mathbf{6 7}$ & $\mathbf{7 1}$ & $\mathbf{7 3}$ & $\mathbf{7 7}$ & $\mathbf{7 8}$ & $\mathbf{7 9}$ \\
\hline 17 & 2 & 1 & 1 & 0 & 2 & 0 & 3 & 1 & 1 & 1 & 4 & 0 & 1 & 2 & 3 & 4 & 0 & 1 & 0 \\
18 & 0 & 1 & 1 & 4 & 3 & 2 & 1 & 1 & 2 & 1 & 1 & 1 & 1 & 1 & 0 & 0 & 1 & 1 & 1 \\
19 & 2 & 1 & 1 & 5 & 1 & 1 & 1 & 5 & 1 & 1 & 3 & 4 & 1 & 1 & 0 & 1 & 2 & 1 & 1 \\
20 & 2 & 1 & 1 & 0 & 1 & 1 & 1 & 6 & 1 & 1 & 3 & 1 & 2 & 2 & 4 & 0 & 1 & 1 & 1 \\
21 & 2 & 1 & 3 & 2 & 3 & 1 & 1 & 1 & 1 & 1 & 1 & 0 & 1 & 1 & 6 & 5 & 1 & 1 & 1 \\
22 & 2 & 1 & 1 & 0 & 0 & 1 & 0 & 5 & 0 & 0 & 1 & 0 & 2 & 1 & 0 & 2 & 0 & 1 & 0 \\
23 & 1 & 1 & 1 & 1 & 0 & 1 & 0 & 1 & 2 & 1 & 3 & 1 & 1 & 2 & 7 & 1 & 1 & 1 & 0 \\
24 & 0 & 1 & 1 & 0 & 0 & 1 & 3 & 2 & 4 & 1 & 3 & 1 & 0 & 2 & 5 & 1 & 2 & 1 & 1 \\
25 & 1 & 1 & 0 & 0 & 1 & 1 & 0 & 4 & 0 & 2 & 1 & 1 & 1 & 1 & 0 & 0 & 1 & 1 & 0 \\
26 & 0 & 1 & 1 & 0 & 0 & 0 & 0 & 2 & 1 & 2 & 1 & 1 & 3 & 0 & 0 & 3 & 2 & 1 & 0 \\
27 & 1 & 1 & 0 & 1 & 2 & 1 & 0 & 2 & 2 & 2 & 2 & 1 & 0 & 0 & 0 & 3 & 0 & 1 & 0 \\
28 & 1 & 4 & 1 & 0 & 3 & 1 & 0 & 2 & 2 & 1 & 2 & 1 & 2 & 2 & 1 & 1 & 0 & 1 & 2 \\
29 & 1 & 1 & 1 & 0 & 0 & 1 & 4 & 4 & 1 & 1 & 3 & 1 & 1 & 2 & 0 & 1 & 1 & 1 & 0 \\
30 & 1 & 1 & 1 & 0 & 1 & 1 & 1 & 2 & 1 & 1 & 1 & 1 & 2 & 1 & 0 & 1 & 1 & 1 & 0 \\
31 & 1 & 4 & 1 & 0 & 0 & 1 & 0 & 2 & 2 & 0 & 1 & 1 & 1 & 0 & 1 & 0 & 1 & 1 & 1 \\
32 & 1 & 1 & 5 & 0 & 0 & 1 & 3 & 4 & 6 & 1 & 2 & 1 & 0 & 2 & 1 & 1 & 1 & 1 & 1 \\
\hline
\end{tabular}

Accessions were marked with their genotype group number after SSR-HRM analyses. "0" means invalid data.

In addition, to evaluate the reliability and efficiency of SSR-HRM method, polyacrylamide gel electrophoresis (PAGE) and sequencing were carried out using the same PCR product obtained with ZJULM 50, thus the results can directly be compared with SSR-HRM results in Figure 6. The results of PAGE could only cluster those 32 accessions to four taxa (Figure 7). All PCR products obtained with ZJULM 50 were sequenced, and, to get the cluster result of these sequencing data, evolutionary relationships of those 32 accessions were measured by MEGA7, based on Neighbor-Joining method [18] (Figure 8).

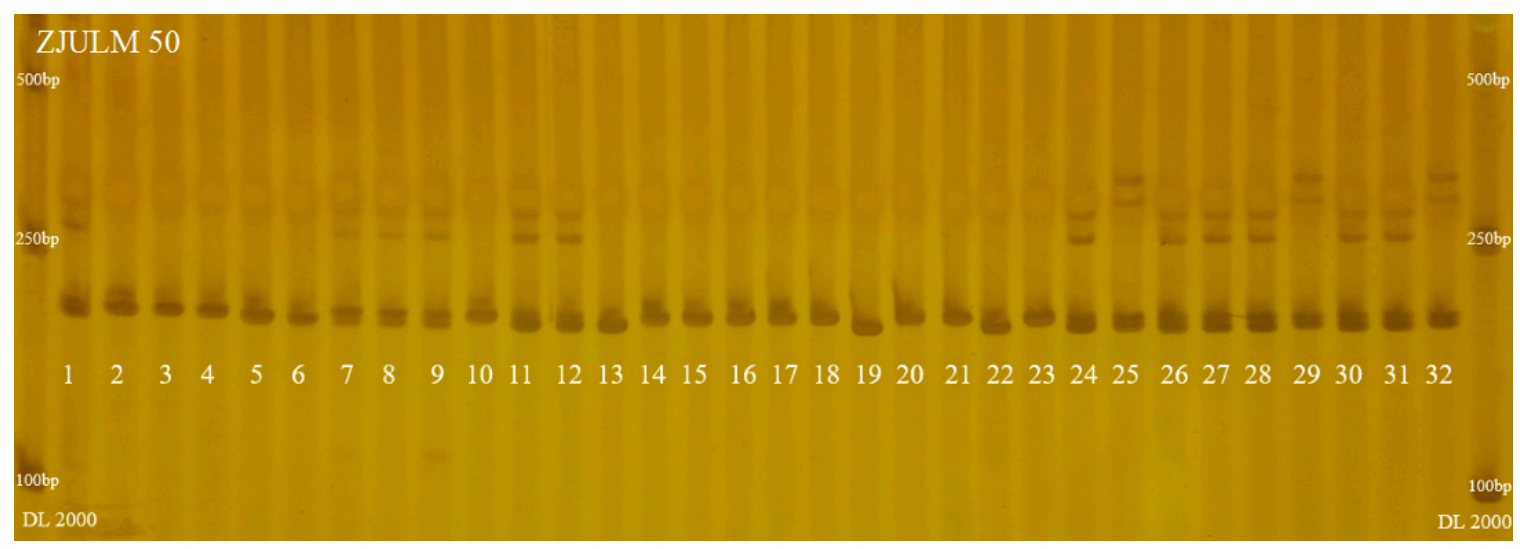

Figure 7. Results of PCR products obtained with the SSR marker ZJULM 50 on $6 \%$ denaturing polyacrylamide gel at $60 \mathrm{~mA}$ constant current (Nos. 1-32, 32 accessions, detailed explanation can be seen in Table 6; DNA Marker: DL2000).

According to these 19 group SSR-HRM genotype data (Table 5), dendrogram for 32 Luffa accessions were performed (Figure 9) based on Nei's genetic distance coefficient [19], and then these 32 accessions were divided into two groups: Cluster A and Cluster B. Cluster A was comprised of 28 accessions belonging to L. cylindrica, and Cluster B included four accessions belonging to L. acutangula. The genetic distance coefficient between L. cylindrica and L. acutangula was 0.11 , higher than 0.00 that was previously reported by $\mathrm{Wu}$ et al. [8]. According to cluster analysis above, the similarity among all these accessions ranged from 0.11 to 0.86 . 


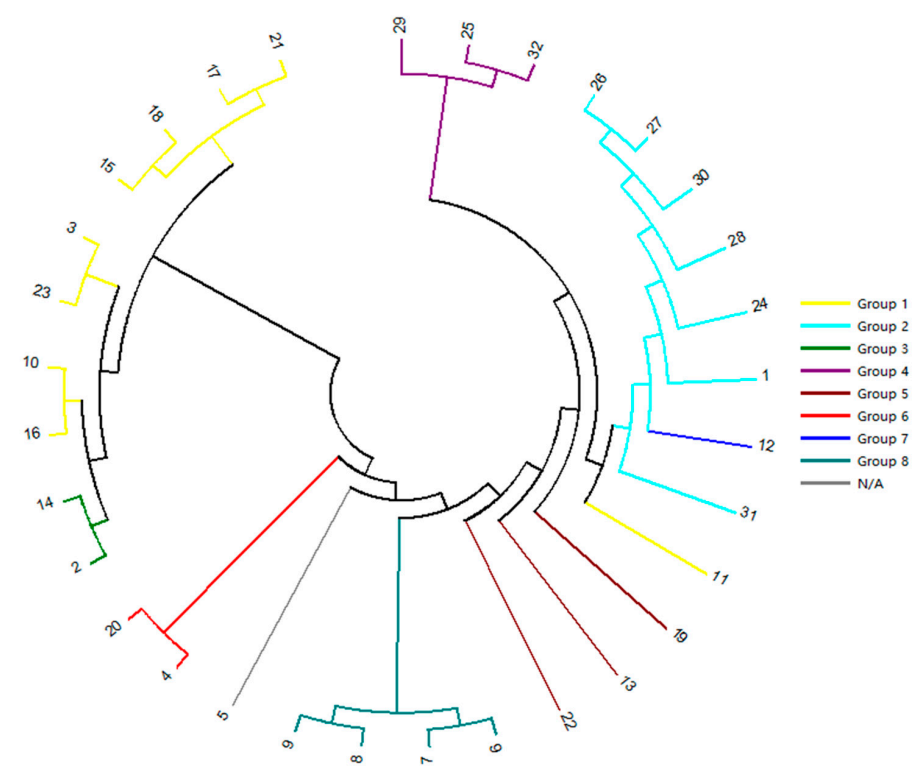

Figure 8. Evolutionary relationships of PCR products obtained with the SSR marker ZJULM 50 based on sequencing analysis. (Nos. 1-32, 32 accessions, detailed explanation can be seen in Table 7; the color of the branches is the same with the result of SSR-HRM group color in Figure 6E).



Figure 9. Dendrogram for 32 Luffa accessions derived from UPGMA cluster analysis obtained with the 19 genomic SSR markers using HRM analysis. Coefficient was based on Nei's genetic distance coefficient [19]. 


\section{Discussion}

\subsection{Characteristics of Luffa cylindrica L. Genome}

The genomes of Cucurbitaceae family members Cucumis melo L. and Cucumis sativus L. have been reported. The genome size of Cucumis melo L. was $375 \mathrm{Mb}$, representing $83.3 \%$ of the estimated melon genome [20], while Cucumis satious L. was only $243.5 \mathrm{Mb}, 72.8 \%$ sequence anchored on chromosome [21]. From our genome survey data, the estimated genome size of L. cylindrica was $789.97 \mathrm{Mb}$ using all of the clean data for K-mer analysis, which was almost two times of Cucumis melo L. and three times of Cucumis sativus L. Genome data of L. cylindrica can be improved by the additional sequencing of larger insert libraries to increase the contig and scaffold sizes. With the development of NGS technologies, genome sequencings of horticultural plants, such as L. cylindrica, not only help easily understand genome organization and critical gene associated with important traits, but also help conveniently design more highly polymorphic molecular markers for subsequent application in molecular breeding [22]. In addition, these data here contribute to genomic research of sponge.

The GC content affected the sequence bias directly [23]. More than $65 \%$ or less than $25 \%$ GC contents might cause sequence bias on the Illumina sequencing platform. Because genomic sequences obtained through high-throughput sequencing are not uniformly distributed across the genome. This systematic bias is a particular problem for techniques, thus seriously affecting genome assembly [24,25]. L. cylindrica had 37.90\% GC content, which was higher than that of potatoes (34.8-36.0\%) [26,27], while lower than that of Rosa roxburghii Tratt $(\sim 38.64 \%)$ [8], Gracilariopsis lemaneiformis ( 48\%) [9], human ( 41\%) and Nasonia vitripennis ( 40.6\%) [28].

From the 1,064,011,890 bp genome survey sequence, 125,094 SSRs without mono-nucleotide repeats were identified. Therefore, the distribution of SSRs in the genome of L. cylindrica was estimated to be about 117.57 SSRs per Mb, which is lower than the 135.5 SSRs per Mb in Arabidopsis [29]. Among the di-nucleotide repeat motifs, AT/AT accounted for $64.56 \%$ and was confirmed to be the most abundant type, followed by AG/CT, accounting for $25.81 \%$. This was consistent with the results that AAT/ATT, AAAT/ATTT, AAAAT/ATTTT and AAAAAG/CTTTTTT were the most abundant repeat motifs in their SSR motif repeats, and they were all A/T rich motifs existing in L. cylindrica. This phenomenon was similar to other species such as Brassica napus [30], rice [31], peanut [32] and Arabidopsis [33], in which A/T rich motifs also performed a dominant role.

\subsection{Genomic SSR Markers Development}

In this study, 65/80 of genomic SSR markers could be amplified by "Zheda 23" (L. cylindrica) and 59/80 by "Zheda 83" (L. acutangula) based on agarose gel electrophoresis. Nineteen markers were used for SSR-HRM, and the other markers (Table 6), which could also be amplified by "Zheda 23" or "Zheda 83", might be meaningful for further study such as QTL mapping. All of these genomic SSR markers were valuable for fingerprinting and genetic analysis of Luffa.

Table 6. Genomic SSR markers designed.

\begin{tabular}{cccc}
\hline Marker & GenBank Accession & $\begin{array}{c}\text { Primer sequence } \\
\left(\mathbf{5}^{\prime}-\mathbf{3}^{\prime} \mathbf{)} \text { (Forward) }\right.\end{array}$ & Primer sequence $\left(\mathbf{5}^{\prime} \mathbf{- 3}^{\prime} \mathbf{)}\right)$ (Reverse) \\
\hline ZJULM 1 & MF677780 & AAATTGGGTATCCATCTC & CATAAAACTTCCGTGAAA \\
ZJULM 2 & MF677781 & TATTTGGTCCAACAATAG & TTGAAAGTTCAATAAACC \\
ZJULM 4 & MF677782 & CTTTGGGCTTCTTCACTA & TTTGGGTGAAAGTTTTGT \\
ZJULM 5 & MF677783 & TCAACACTCTGCCAATTG & AGCCCCATGAACATAAAA \\
ZJULM 6 & MF677784 & TAAAAGTTCATTCATTACAC & GACATAACAAAATAGGATAA \\
ZJULM 7 & MF677785 & ATCTAAAATAAATTAACGGG & CAAATTTGGTTGAATTTACA \\
ZJULM 8 & MF677786 & ATTTGATTCGATGCTACC & GAGCTCTTCGGAATTTTA \\
ZJULM 9 & MF677787 & AAAGAAGCATAATCCCTT & TAACCTGCAATTCAATGT \\
ZJULM 10 & MF677788 & ATGGGAGTTGGGCTATTT & ATCAGCAGCAGTGTTTGG \\
ZJULM 11 & MF677789 & TCTTCCTCCCTCTTATCC & TCTGAATGGGGTTGGTTT \\
ZJULM 12 & MF677790 & GTGTTTGGTTATGAATTT & CCTTATAATTTCAATTCC \\
\hline
\end{tabular}


Table 6. Cont.

\begin{tabular}{|c|c|c|c|}
\hline Marker & GenBank Accession & $\begin{array}{l}\text { Primer sequence } \\
\left(5^{\prime}-3^{\prime}\right)(\text { Forward) }\end{array}$ & Primer sequence $\left(5^{\prime}-3^{\prime}\right)($ Reverse $)$ \\
\hline ZJULM 13 & MF677791 & TCTСССТСССТСТTGСТC & TGTAAACTTAACCCAAACCTC \\
\hline ZJULM 14 & MF677792 & ТTCТTCTCAGGCACTCСA & CACAAAGTACCAAGGTGG \\
\hline ZJULM 15 & MF677793 & TGTTTGGATCTAAAGAAA & TAAACAACATGGATGAAT \\
\hline ZJULM 16 & MF677794 & CTCTATGAGGTCTGTGGGAGA & GAGCTAAGCCCCAAAATC \\
\hline ZJULM 17 & MF677795 & TTGTCTTTACTATTGGGA & АTТАТСАААСАТССАСАА \\
\hline ZJULM 18 & MF677796 & TCAGGATTGTTAAGCCAGTT & CAATGACCAGCAATGACC \\
\hline ZJULM 19 & MF677797 & GCACCTAAGCCAACCAAC & GGACAATGCATGTCACGA \\
\hline ZJULM 22 & MF677798 & GAGAAGAAGACTCTGGGG & AAGAAAAGTGAAATCCCA \\
\hline ZJULM 23 & MF677799 & GAAAAGTCGTTGACAACA & CAATTTCGTTTGAATGTT \\
\hline ZJULM 24 & MF677800 & CGAATGTTAAAGAAACTT & CGAATGTTAAAGAAACTT \\
\hline ZJULM 25 & MF677801 & AAAAGTCGTTGACAACAT & TACAATTTCGTTTGAATG \\
\hline ZJULM 26 & MF677802 & AAACAGTTTCССТTACCA & AATATCGTGGAGGTTGTC \\
\hline ZJULM 27 & MF677803 & GGACACCAAAGTAAACATGC & CTAGTTTCATCAATTCCAAG \\
\hline ZJULM 28 & MF677804 & ACTTGCTTATCAGAGTGGCA & ATGTTTGTCGGTAATGTTCG \\
\hline ZJULM 29 & MF677805 & CCACCTGTAATGTTATCCAT & ATTTTGGTACGTTATCTGCT \\
\hline ZJULM 30 & MF677806 & AGCAACTAAAATGAGGTAAA & TATTGATGGCATCCATCCTG \\
\hline ZJULM 31 & MF677807 & GCCAACTCATAACAAGAATC & TAATCACCAACACCTTATTC \\
\hline ZJULM 32 & MF677808 & GAAATGTGAAATCCCACG & TGGACGGAGTAGAGGTGA \\
\hline ZJULM 33 & MF677809 & TAGCCGTTCGTTTTCATT & CACCGACATTCTAAATCCTG \\
\hline ZJULM 34 & MF677810 & CATGGCGGCTATGAAGGC & TCCGCACAGTGACAGAGTGGT \\
\hline ZJULM 35 & MF677811 & TTATGTCTGTCCCGTTCA & ATACСТTATCTTTGTGCC \\
\hline ZJULM 38 & MF677812 & GGGGAGAAATAAGAAATAG & TTCGCTTCGTGGTGTTGG \\
\hline ZJULM 40 & MF677813 & TATCCAATAAGCTTGAAG & AAACTATCGCATGTAATG \\
\hline ZJULM 43 & MF677814 & CTACCCGTGAGAATTTGA & CACTACTТССАСССАСАA \\
\hline ZJULM 48 & MF677815 & TAGAAAGGAAAGGAGGAA & TTCAAGAGTTCAGGGTTT \\
\hline ZJULM 49 & MF677816 & GAGAAAGATAATTGAAAGGGAT & Г GTGCTGCCATACGGTTAG \\
\hline ZJULM 52 & MF677817 & ATCTAAAATTTAAAGGGG & TAGACCATAATACCCСТT \\
\hline ZJULM 54 & MF677818 & TGTTGTTATGAATCGGTGAA & TAGGCAAAGGAAAGTTGG \\
\hline ZJULM 56 & MF677819 & TGGCGGCGGAGCAGTGAA & ACCACCCGTAGGGCGTGTCC \\
\hline ZJULM 57 & MF677820 & ТTСТTСТСССТСТTТGСТ & ACAGTCACCGCCTCATAT \\
\hline ZJULM 58 & MF677821 & GTATCGTATCGGGTGCCT & ТТССТТТССАСАТGССТС \\
\hline ZJULM 60 & MF677822 & ATTTCTGTTAATTTGGTTCC & CAATCGAATAAAAGGTCAA \\
\hline ZJULM 62 & MF677823 & TTTTCAAAGTTCAAGGAC & TTAGTGTCACGTCAGCAT \\
\hline ZJULM 63 & MF677824 & CAGGCGAAGCAAAGGATT & TGATGGTCTGACGGAGGC \\
\hline ZJULM 64 & MF677825 & TTTGTCACAATCCCACCT & GAATACGCAGCCTTCTTT \\
\hline ZJULM 65 & MF677826 & AGAATGATTTACCCGTAG & AGAGGAGGAACTTTTGAT \\
\hline ZJULM 68 & MF677827 & ССССТССССТССААААТА & TTGCCCAGGAACGAACTT \\
\hline ZJULM 69 & MF677828 & TCATTCCTACCGAAAGTA & AACGGACCCTTATACTTG \\
\hline ZJULM 70 & MF677829 & AAGCGGGAGCTAAGAATG & GCTGGAATGTTGGGAGAA \\
\hline ZJULM 72 & MF677830 & ACACCGTAACAGATCAAA & СТСАТТСТТТСССТТТСТ \\
\hline ZJULM 74 & MF677831 & ATCTAAAATTTAAAGGGG & TAGACCATAATACCCCTT \\
\hline ZJULM 75 & MF677832 & TGTTGTTATGAATCGGTGAA & TAGGCAAAGGAAAGTTGG \\
\hline ZJULM 76 & MF677833 & AACCCACAGAATAAAGATG & GAAGAAGCTCCTACCTGA \\
\hline ZJULM 80 & MF677834 & TCAATGCCAGTGTCTCAA & GCTTCTTATTGGACCTATTT \\
\hline
\end{tabular}

\subsection{Genetic Relationship Analysis by SSR-HRM}

Following the standard protocol of HRM, experiment data were obtained that could be analyzed by corresponding software. During analysis period, deltaTm discrimination and curve shape sensitivity were two core parameters that significantly affected the clustering results. If the sensitivity were too high, the clustering results obtained with the same SSR marker would be diverse from each other, while, if the sensitivity were too low, it would lead to completely consistent results. These two situations would make clustering difficult to carry out. According to the experiment, $50 \%$ would be the appropriate level of these two parameters. In this case, the curves were analyzed, melting curves and difference plots were generated, and these curves were clustered to several genotype groups. The corresponding accessions was recorded with their genotype group number, and then these genotype results were analyzed with traditional SSR analysis software, such as PopGene, Power Marker, NTSYS, etc. It provided us an unusual experience of quickly identifying different accessions.

PAGE and sequencing are traditional methods to analyze PCR products. From the results of PCR products obtained with ZJULM 50 based on PAGE (Figure 7), these 32 accessions could be 
clustered into four taxa, while SSR-HRM (Figure 6) easily gathered those accessions into taxa with visual difference. As for the result of sequencing (Figure 8), more than nine taxa were demonstrated.

Comparing the result of SSR marker ZJULM 50 by PAGE, sequencing and SSR-HRM methods, many similarities and differences were found. For example, Nos. 25, 29 and 32 had a similar banding pattern in PAGE, and were also proven to be closely related in sequencing and SSR-HRM. A similar situation was found in Nos. 13, 19 and 22 accessions. In addition, Nos. 1, 7, 8, 9, 11, 12, 24, 26, 27, 28, 30 and 31 in PAGE have similar banding patterns, with one main belt and two incidental ones represented at the same level, thus these accessions were classified to one taxon. However, according to the sequencing result (Figure 8), Nos. 7-9 were clearly separated from the other nine accessions. This result was closer to the sample characteristics that Nos. 7-9 accessions were L. acutangula, but the other nine accessions belonged to L. cylindrica. The result of SSR-HRM (Figure 6E) was nearly the same with sequencing on this point, in which Nos. 1, 24, 26, 27, 28, 30 and 31 were identified to be same taxon, and Nos. 7-9 were classified to another taxon. In addition, as No. 6 belonged to L. acutangula, No. 6 showed a relatively close genetic relationship with Nos. 7-9 in sequencing and SSR-HRM. However, different banding patterns obviously misled the judgment of No. 6 in PAGE. Thus, it suggested that SSR-HRM could distinguish better and was much more accurate than PAGE. Based on different calculating method, sensibility and identifying principles, the result of SSR-HRM derived from one pair of SSR markers might different from sequencing, such as Nos. 11 and 12 accessions. However, the final result of the genetic relationship would tend to be consistent with the increasing number of markers used.

In total, 135 primer pairs were used to analyze the genetic relationship among 32 Chinese bayberry (Myrica rubra) accessions through PAGE method [7]. However, 32 Luffa specific cultivars could easily be identified by only 19 SSR markers in this study. It indicated that SSR-HRM is a method with relative high resolution, high throughput and efficiency. Compared with sequencing, SSR-HRM requires less money and could obtain nearly the same result in a relatively short period. Therefore, SSR-HRM has become increasingly popular in many crop analyses such as cultivar identification and genotyping $[11,15,16]$.

In this study, 28 accessions belonging to L. cylindrica and four accessions belonging to L. acutangula were clearly divided into two clusters (Figure 9). Cluster A was L. cylindrica with different phenotypes. Both "Lvbaoshi" and "Chunjianlv" were from Lanzhou, Gansu Province. They had high genetic distance coefficient $(\sim 0.83)$, which was mainly relevant with their highly similar phenotype, such as $200-400 \mathrm{~g}$ weight, green fruit color, $4 \mathrm{~cm}$ diameter and $35-40 \mathrm{~cm}$ length per sponge fruit. In addition, "Jipinduanbang" was green color, short length $(\sim 18 \mathrm{~cm})$ and thick diameter $(\sim 6.5 \mathrm{~cm})$, while "Baimeichunxiang" was almost white color, $\sim 24 \mathrm{~cm}$ length and about $6 \mathrm{~cm}$ diameter. Thus, they were clearly separated and genetic distance coefficient was only $~ 0.59$. All four cultivars in Cluster B belonged to L. acutangula. "Shuangjiannaihan" was from Guangdong Province; however, it had $\sim 0.71$ genetic distance coefficient with the local cultivar "Zheda 84 ". It suggested that the similar genetic characteristics existed in those two cultivars. The coefficient ranged from 0.11 to 0.86 throughout the result of dendrogram, and the accessions with similar traits were clustered together. Therefore, based on the advantages of HRM and genomic SSR markers, SSR-HRM technology was considered as a rapid, cost effective and high-throughput method for genotyping analyses.

\section{Materials and Methods}

\subsection{Plant Materials and DNA Extraction}

Luffa cultivar "Zheda 23" (Luffa cylindrica L.) was grown in the Zijingang Campus, Zhejiang University, Hangzhou City, China $\left(30^{\circ} 18^{\prime} 18^{\prime \prime} \mathrm{N}, 120^{\circ} 4^{\prime} 44.4^{\prime \prime} \mathrm{E}\right)$ for the genome survey, which possessed the characteristic of typical cultivation. The genomic DNA was extracted from the tender leaves using the DNA Kit (Tiangenbiotech, Beijing, China). The quality and amount of DNA were checked by means of spectrophotometer analysis using Nanodrop 2000. In addition, the DNA of 32 cultivars (Table 7) was also extracted from the tender leaves by DNA kit (Foregene, Chengdu, China). 
Table 7. The 32 Luffa accessions included in present study.

\begin{tabular}{cccccc}
\hline No. & Accession & Region & No. & Accession & Region \\
\hline 1 & Zheda 1 & Hangzhou, Zhejiang & 17 & Zheda 7 & Hangzhou, Zhejiang \\
2 & Zheda 2 & Hangzhou, Zhejiang & 18 & Zheda 8 & Hangzhou, Zhejiang \\
3 & Zheda 23 & Hangzhou, Zhejiang & 19 & Zheda h101 & Hangzhou, Zhejiang \\
4 & Zheda 56 & Hangzhou, Zhejiang & 20 & Zheda h100 & Hangzhou, Zhejiang \\
5 & Zheda 10 & Hangzhou, Zhejiang & 21 & Zheda 57N & Hangzhou, Zhejiang \\
6 & Zheda 81 & Hangzhou, Zhejiang & 22 & Zheda 72 & Hangzhou, Zhejiang \\
7 & Zheda 83 & Hangzhou, Zhejiang & 23 & Zheda 22 & Hangzhou, Zhejiang \\
8 & Zheda 84 & Hangzhou, Zhejiang & 24 & Zheda 70 & Hangzhou, Zhejiang \\
9 & Shuangjiannaihan1 & Heshan, Guangdong & 25 & Zheda 5 & Hangzhou, Zhejiang \\
10 & Jizaoxiangyu & Changsha, Hunan & 26 & Zheda h6 & Hangzhou, Zhejiang \\
11 & Jinxiuzaojia & Lanzhou, Gansu & 27 & Zheda h7 & Hangzhou, Zhejiang \\
12 & Sanbier & Changde, Hunan & 28 & Zheda 85 & Hangzhou, Zhejiang \\
13 & Baimeichunxiang & Changsha, Hunan & 29 & Zheda 86 & Hangzhou, Zhejiang \\
14 & Jipinduanbang & Changsha, Hunan & 30 & Zheda 87 & Hangzhou, Zhejiang \\
15 & Lvbaoshi & Lanzhou, Gansu & 31 & Zheda 88 & Hangzhou, Zhejiang \\
16 & Chunjianlv & Lanzhou, Gansu & 32 & Zheda h4 & Hangzhou, Zhejiang \\
\hline
\end{tabular}

\subsection{Genome Sequencing and Sequence Assembly}

Following the standard protocol (Illumina, Beijing, China), DNA library with insert size of 220 base pairs (bp) was constructed from randomly fragmented genomic DNA. Sequencing date was produced using the Illumina HiSeq 2500 sequencing platform (Beijing Biomarker Technologies Co., Ltd. Beijing, China). After filtering and correction of the raw data, clean reads were obtained. The high quality reads were then assembled to contigs and scaffolds using SOAPdenovo software (http://soap.genomics.org.cn/soapdenovo.html). All of the clean reads were used to conduct de novo assembly.

\subsection{Genome Size Estimation, GC Content and Genome Survey}

All of the clean data were used for K-mer analysis. K-mer analysis was used to estimate the genome size (Genome size $=\mathrm{K}$-mer count/Peak of the depth distribution) and characters, such as repetitive sequences and heterozygosis. The GC average sequencing depth and content was calculated by the 10-kb non-overlapping sliding windows along the assembled sequence. The characteristics of SSR motif were briefly shown according to the genome survey.

\subsection{Genomic SSR Marker Development}

The Perl script microsatellite searching tool (MISA) (http://pgrc.ipk-gatersleben.de/misa/misa. $\mathrm{html}$ ) was used to identify microsatellite repeats in L. cylindrica genome sequence database. In this study, the SSR loci containing perfect SSR motif repeats of 2-6 nucleotides were only considered. The minimum SSR length criteria were defined as six reiterations for di-nucleotide, and five reiterations for other SSR motif repeats. Eighty primers were designed by Primer Premier 5.0 (Premier Biosoft International, Palo Alto, CA, USA), and all followed the parameters: 18-22 bp primer size, 100-300 bp product length and $50-60^{\circ} \mathrm{C}$ annealing temperature. Primers were synthesized by TsingKe Co., Ltd., Hangzhou, China.

\subsection{Genetic Relationship Analysis by SSR-HRM}

We primarily tested two Luffa cultivars ("Zheda 23" and "Zheda 83") for 80 SSR loci. SSR PCR amplification was conducted in a $20 \mu \mathrm{L}$ volume containing $10 \mathrm{ng}$ of genomic DNA, $2 \mathrm{U}$ Taq DNA polymerase (TaKaRa, Dalian, China), $2.0 \mathrm{mM} \mathrm{MgCl}_{2}, 0.20 \mathrm{mM}$ dNTPs and $0.2 \mu \mathrm{M}$ each primer. The PCR protocol consisted of a pre-incubation at $95^{\circ} \mathrm{C}$ for $300 \mathrm{~s}$, followed by 45 cycles of $95^{\circ} \mathrm{C}$ for $10 \mathrm{~s}$, annealing for $10 \mathrm{~s}$ at $50^{\circ} \mathrm{C}, 72{ }^{\circ} \mathrm{C}$ for $10 \mathrm{~s}$, and a final extension step of $72{ }^{\circ} \mathrm{C}$ for $10 \mathrm{~min}$. PCR reactions were carried out in a thermal cycler C1000 (Bio-Rad, Hercules, California, USA). PCR products were separated on 3\% 
agarose gels at $110 \mathrm{~V}$, then stained with GelRedTM (Biotium, Hayward, CA, USA) and photographed under UV light using Image LabTM software Version 2.0.1 (Bio-Rad, Hercules, CA, USA).

To evaluate the reliability and efficiency of SSR-HRM method, PAGE and sequencing were carried out. As for PAGE, PCR products obtained with the SSR marker ZJULM 50 were run on $6 \%$ denaturing polyacrylamide gel at $60 \mathrm{~mA}$ constant current and polymorphism was detected by silver staining [34]. GelAnalyzer (Version 2010a) was used to analyze the gel image generated by PAGE. Sequencing of PCR products obtained with the SSR marker ZJULM 50 were sequenced by ABI 3730xl sequencing platform (TsingKe Co., Ltd., Hangzhou, China). The evolutionary relationships analysis (Figure 8) was conducted by Molecular Evolutionary Genetics Analysis version 7.0 (MEGA7). The evolutionary history was inferred using the Neighbor-Joining method [18]. The bootstrap consensus tree inferred from 10,000 replicates was taken to represent the evolutionary history of the taxa analyzed [35]. Branches corresponding to partitions reproduced in less than $50 \%$ bootstrap replicates are collapsed. The evolutionary distances were computed using the Maximum Composite Likelihood method [36] and are in the units of the number of base substitutions per site. Codon positions included were 1st + $2 \mathrm{nd}+3 \mathrm{rd}+$ Noncoding. All positions containing gaps and missing data were eliminated. Evolutionary analyses were conducted in MEGA7 [37].

Nineteen distinct genomic SSR markers were chosen to identify 32 cultivars by Real-Time PCR system using SSR High Resolution Melting method (SSR-HRM). The high resolution melting was carried out by the LightCycler 96 real Real-Time polymerase chain reaction (PCR) System (Roche Diagnostics GmbH, Roche Applied Science, Mannheim, Germany) in a total volume of $20 \mu \mathrm{L}$, which containing $10 \mathrm{ng}$ of genomic DNA, $2.0 \mathrm{mM} \mathrm{MgCl}_{2}, 10 \mu \mathrm{L} \mathrm{LightCycler}^{\circledR} 480$ High Resolution Melting Master Mix with LightCycler ${ }^{\circledR} 480$ ResoLight Dye (Roche, Basel, Switzerland), $0.2 \mu$ M reverse primer and $0.2 \mu \mathrm{M}$ forward primer. The reactions were subjected to a touchdown PCR thermal protocol consisting of an initial incubation $95^{\circ} \mathrm{C}$ for $600 \mathrm{~s}$, then followed by 55 cycles at $95^{\circ} \mathrm{C}(20 \mathrm{~s}), 62-50{ }^{\circ} \mathrm{C}$ (20 s), and $72{ }^{\circ} \mathrm{C}(20 \mathrm{~s})$. The annealing temperature decreased by $1{ }^{\circ} \mathrm{C}$ per cycle from 62 to $50{ }^{\circ} \mathrm{C}$, and then kept $50{ }^{\circ} \mathrm{C}$ in subsequent cycle. The amplification procedure was immediately followed by the high resolution melting steps: $95^{\circ} \mathrm{C}$ for $60 \mathrm{~s}$, cooling to $40{ }^{\circ} \mathrm{C}$ for $60 \mathrm{~s}$, and then the temperature was rapidly raised to $65^{\circ} \mathrm{C}$. Subsequently, the temperature was raised from 65 to $97^{\circ} \mathrm{C}$ with each steps of $0.02{ }^{\circ} \mathrm{C}$ for $1 \mathrm{~s}$. After reactions, SSR-HRM raw data were obtained.

LightCycler ${ }^{\circledR} 96$ (Version 1.1.0.1320 Roche Applied Science, Mannheim, Germany) was used to analyze the SSR-HRM raw data. In the analysis option, both deltaTm discrimination and curve shape sensitivity were set up to $50 \%$ and afterwards the curves were analyzed, then melting curves and difference plots were generated, and finally these curves would be clustered to several genotype groups. The genotype result needed be list strictly with their accessions and markers (Table 5). Each HRM reaction was three repeats and some reactions might fail to perform a HRM process, then some of wrong data would be generated. These wrong data strongly affected the judgment of normal data. Therefore, these wrong data must be removed, and the corresponding genotype number should be encode " 0 ". Then, the genotype results could be analyzed by traditional software. For example, Nei's (1973) gene diversity, Polymorphism information content (PIC) was calculated using Power Marker (version 3.25) [38] (http://statgen.ncsu.edu/powermarker/downloads.htm). The genetic similarity coefficient and UPGMA cluster analysis of these accessions (Figure 9) were calculated by NTSYS software (Version 2.10e, New York, NY, USA) [39].

\section{Conclusions}

Luffa cylindrica (L.) Roem. had a genome of about $789.97 \mathrm{Mb}$ with little heterozygosity. The genomic SSR combined high resolution melting could be effectively used for genotype relationship analysis of Luffa species.

Supplementary Materials: All SSR Markers developed based on de novo genome assembly sequence of L. cylindrica have been deposited in LabArchives at http://dx.doi.org/10.6070/H4QF8QZQ. All SSR-HRM result, including normalized melting curves, different plots and genotype group result have also been deposited in 
LabArchives at http:/ /dx.doi.org/10.6070/H4KS6PNQ. All other data supporting the conclusions of this article are included within the article and its additional files.

Acknowledgments: This research was supported by the National Natural Science Foundation of China (Nos. 31371708, 31201279, and 31671774), Zhejiang Provincial Natural Science Foundation (Nos. LY15C130002 and LZ14C130002), the Fundamental Research Funds for the Central Universities (No. 2015QNA6019), Dabeinong Funds for Discipline Development and Talent Training in Zhejiang University and Jiangsu Collaborative Innovation Center for Modern Crop Production, China.

Author Contributions: Conceived and designed the experiments: Yajing Guan, Jin Hu and Jianyu An; Performed the experiments: Jianyu An, Qin Zhang, Mengqi Yin, Xiaowen Jia and Dongting Gong; Analyzed the data: Jianyu An and Yajing Guan; Contributed reagents/materials/analysis tools: Jianyu An and Qin Zhang; Wrote the paper: Jianyu An and Yajing Guan. All authors have read and approved the manuscript.

Conflicts of Interest: The authors declare no conflict of interest.

\section{Abbreviations}

$\begin{array}{ll}\text { SSR-HRM } & \text { Microsatellite high resolution melting } \\ \text { NGS } & \text { Next-generation sequencing } \\ \text { SSR } & \text { Microsatellite } \\ \text { UPGMA } & \text { Unweighted pair group method analysis } \\ \text { EST } & \text { Expressed sequence tag } \\ \text { ZJULM } & \text { Zhejiang University luffa marker } \\ \text { MISA } & \text { Microsatellite searching tool } \\ \text { GC } & \text { Guanine pluscytosine } \\ \text { NCBI } & \text { National center for biotechnology information } \\ \text { HRM } & \text { High resolution melting } \\ \text { PCR } & \text { Polymerase chain reaction } \\ \text { MEGA } & \text { Molecular evolutionary genetics analysis } \\ \text { PIC } & \text { Polymorphism information content } \\ \text { PAGE } & \text { Polyacrylamide gel electrophoresis }\end{array}$

\section{References}

1. Wu, H.B.; He, X.L.; Gong, H.; Luo, S.B.; Li, M.Z.; Chen, J.Q.; Zhang, C.Y.; Yu, T.; Huang, W.P.; Luo, J.N. Genetic linkage map construction and QTL analysis of two interspecific reproductive isolation traits in sponge gourd. Front. Plant. Sci. 2016, 7. [CrossRef] [PubMed]

2. Joshi, B.K.; Kc, H.B.; Tiwari, R.K.; Ghale, M.; Sthapit, B.R. Descriptors for sponge gourd (Luffa cylindrica (L.) Roem.). Narc Kathmandu Np; 2004. Available online: https://idl-bnc-idrc.dspacedirect.org/bitstream/ handle/10625/31459/122785.pdf?sequence=1.

3. Partap, S.; Kumar, A.; Sharma, N.K.; Jha, K.K. Luffa cylindrica: An important medicinal plant. J. Nat. Prod. Plant Resour. 2012, 2, 127-134.

4. Sheng, Z.; Jin, H.; Zhang, C.F.; Guan, Y.J.; Ying, Z. Genetic analysis of fruit shape traits at different maturation stages in sponge gourd. J. Zhejiang Univ. Sci. B 2007, 8, 338-344.

5. Lu, M.; An, H.M.; Li, L.L. Genome survey sequencing for the characterization of the genetic background of Rosa roxburghii tratt and leaf ascorbate metabolism genes. PLoS ONE 2016, 11. [CrossRef] [PubMed]

6. Zhou, W.; Hu, Y.Y.; Sui, Z.H.; Fu, F.; Wang, J.G.; Chang, L.P.; Guo, W.H.; Li, B.B. Genome survey sequencing and genetic background characterization of Gracilariopsis lemaneiformis (Rhodophyta) based on next-generation sequencing. PLOS ONE 2013, 8. [CrossRef] [PubMed]

7. Jiao, Y.; Jia, H.M.; Li, X.W.; Chai, M.L.; Jia, H.J.; Chen, Z.; Wang, G.Y.; Chai, C.Y.; van de Weg, E.; Gao, Z.S. Development of simple sequence repeat (SSR) markers from a genome survey of Chinese bayberry (Myrica rubra). BMC Genom. 2012, 13. [CrossRef] [PubMed]

8. Wu, H.B.; Gong, H.; Liu, P.; He, X.L.; Luo, S.B.; Zheng, X.M.; Zhang, C.Y.; He, X.M.; Luo, J.N. Large-scale development of EST-SSR markers in sponge gourd via transcriptome sequencing. Mol. Breed. 2014, 34, 1903-1915. [CrossRef]

9. Wang, Y.W.; Samuels, T.D.; Wu, Y.Q. Development of 1,030 genomic SSR markers in switchgrass. Theor. Appl. Genet. 2011, 122, 677-686. [CrossRef] [PubMed]

10. Wilhelm, J.; Pingoud, A.; Hahn, M. Validation of an algorithm for automatic quantification of nucleic acid copy numbers by real-time polymerase chain reaction. Anal. Biochem. 2003, 317, 218-225. [CrossRef] 
11. Ganopoulos, I.; Argiriou, A.; Tsaftaris, A. Microsatellite high resolution melting (SSR-HRM) analysis for authenticity testing of protected designation of origin (PDO) sweet cherry products. Food Control 2011, 22, 532-541. [CrossRef]

12. Wittwer, C.T. High-resolution DNA melting analysis: Advancements and limitations. Hum. Mutat. 2009, 30, 857-859. [CrossRef] [PubMed]

13. Wittwer, C.T.; Reed, G.H.; Gundry, C.N.; Vandersteen, J.G.; Pryor, R.J. High-resolution genotyping by amplicon melting analysis using LCGreen. Clin. Chem. 2003, 49, 853-860. [CrossRef] [PubMed]

14. Tindall, E.A.; Petersen, D.C.; Woodbridge, P.; Schipany, K.; Hayes, V.M. Assessing high-resolution melt curve analysis for accurate detection of gene variants in complex DNA fragments. Hum. Mutat. 2009, 30, 876-883. [CrossRef] [PubMed]

15. Bosmali, I.; Ganopoulos, I.; Madesis, P.; Tsaftaris, A. Microsatellite and DNA-barcode regions typing combined with high resolution melting (HRM) analysis for food forensic uses: A case study on lentils (lens culinaris). Food Res. Int. 2012, 46, 141-147. [CrossRef]

16. Xanthopoulou, A.; Ganopoulos, I.; Koubouris, G.; Tsaftaris, A.; Sergendani, C.; Kalivas, A.; Madesis, P. Microsatellite high-resolution melting (SSR-HRM) analysis for genotyping and molecular characterization of an Olea europaea germplasm collection. Plant Genet. Resour. 2014, 12, 273-277. [CrossRef]

17. Chitsaz, H.; Yee-Greenbaum, J.L.; Tesler, G.; Lombardo, M.J.; Dupont, C.L.; Badger, J.H.; Novotny, M.; Rusch, D.B.; Fraser, L.J.; Gormley, N.A.; et al. Efficient de novo assembly of single-cell bacterial genomes from short-read data sets. Nat. Biotechnol. 2011, 29, 915-921. [CrossRef] [PubMed]

18. Saitou, N.; Nei, M. The neighbor-joining method-A new method for reconstructing phylogenetic trees. Mol. Biol. Evol. 1987, 4, 406-425. [PubMed]

19. Nei, M. Genetic distance between populations. Am. Nat. 1972, 106, 283-292. [CrossRef]

20. Garcia-Mas, J.; Benjak, A.; Sanseverino, W.; Bourgeois, M.; Mir, G.; Gonzalez, V.M.; Henaff, E.; Camara, F.; Cozzuto, L.; Lowy, E.; et al. The genome of melon (Cucumis melo L.). Proc. Natl. Acad. Sci. USA 2012, 109, 11872-11877. [CrossRef] [PubMed]

21. Huang, S.W.; Li, R.Q.; Zhang, Z.H.; Li, L.; Gu, X.F.; Fan, W.; Lucas, W.J.; Wang, X.W.; Xie, B.Y.; Ni, P.X.; et al. The genome of the cucumber, Cucumis sativus L. Nat. Genet. 2009, 41, 1275-1281. [CrossRef] [PubMed]

22. Hou, S.Y.; Sun, Z.X.; Bin, L.H.; Xu, D.M.; Wu, B.; Zhang, B.; Wang, X.C.; Han, Y.H.; Zhang, L.J.; Qiao, Z.J.; et al. Genetic diversity of buckwheat cultivars (Fagopyrum tartaricum gaertn.) assessed with SSR markers developed from genome survey sequences. Plant Mol. Biol. Rep. 2016, 34, 233-241. [CrossRef]

23. Cheung, M.S.; Down, T.A.; Latorre, I.; Ahringer, J. Systematic bias in high-throughput sequencing data and its correction by beads. Nucleic Acids Res. 2011, 39. [CrossRef] [PubMed]

24. Aird, D.; Ross, M.G.; Chen, W.S.; Danielsson, M.; Fennell, T.; Russ, C.; Jaffe, D.B.; Nusbaum, C.; Gnirke, A. Analyzing and minimizing PCR amplification bias in illumina sequencing libraries. Genome Biol. 2011, 12. [CrossRef] [PubMed]

25. Bentley, D.R.; Balasubramanian, S.; Swerdlow, H.P.; Smith, G.P.; Milton, J.; Brown, C.G.; Hall, K.P.; Evers, D.J.; Barnes, C.L.; Bignell, H.R.; et al. Accurate whole human genome sequencing using reversible terminator chemistry. Nature 2008, 456, 53-59. [CrossRef] [PubMed]

26. Hirakawa, H.; Okada, Y.; Tabuchi, H.; Shirasawa, K.; Watanabe, A.; Tsuruoka, H.; Minami, C.; Nakayama, S.; Sasamoto, S.; Kohara, M.; et al. Survey of genome sequences in a wild sweet potato, Ipomoea trifida (H.B.K.) G. Don. DNA Res. 2015, 22, 171-179. [CrossRef] [PubMed]

27. Xu, X.; Pan, S.K.; Cheng, S.F.; Zhang, B.; Mu, D.S.; Ni, P.X.; Zhang, G.Y.; Yang, S.; Li, R.Q.; Wang, J.; et al. Genome sequence and analysis of the tuber crop potato. Nature 2011, 475, 189-194. [CrossRef] [PubMed]

28. Werren, J.H.; Richards, S.; Desjardins, C.A.; Niehuis, O.; Gadau, J.; Colbourne, J.K.; Beukeboom, L.W.; Desplan, C.; Elsik, C.G.; Grimmelikhuijzen, C.J.P.; et al. Functional and evolutionary insights from the genomes of three parasitoid Nasonia species. Science 2010, 327, 343-348. [CrossRef] [PubMed]

29. Zhao, H.S.; Yang, L.; Peng, Z.H.; Sun, H.Y.; Yue, X.H.; Lou, Y.F.; Dong, L.L.; Wang, L.L.; Gao, Z.M. Developing genome-wide microsatellite markers of bamboo and their applications on molecular marker assisted taxonomy for accessions in the genus Phyllostachys. Sci. Rep. 2015, 5. [CrossRef] [PubMed]

30. Shi, J.Q.; Huang, S.M.; Zhan, J.P.; Yu, J.Y.; Wang, X.F.; Hua, W.; Liu, S.Y.; Liu, G.H.; Wang, H.H. Genome-wide microsatellite characterization and marker development in the sequenced Brassica crop species. DNA Res. 2014, 21, 53-68. [CrossRef] [PubMed] 
31. Temnykh, S.; DeClerck, G.; Lukashova, A.; Lipovich, L.; Cartinhour, S.; McCouch, S. Computational and experimental analysis of microsatellites in rice (Oryza sativa L.): Frequency, length variation, transposon associations, and genetic marker potential. Genome Res. 2001, 11, 1441-1452. [CrossRef] [PubMed]

32. Zhou, X.J.; Dong, Y.; Zhao, J.J.; Huang, L.; Ren, X.P.; Chen, Y.N.; Huang, S.M.; Liao, B.S.; Lei, Y.; Yan, L.Y.; et al. Genomic survey sequencing for development and validation of single-locus SSR markers in peanut (Arachis hypogaea L.). BMC Genom. 2016, 17. [CrossRef] [PubMed]

33. Katti, M.V.; Ranjekar, P.K.; Gupta, V.S. Differential distribution of simple sequence repeats in eukaryotic genome sequences. Mol. Biol. Evol. 2001, 18, 1161-1167. [CrossRef] [PubMed]

34. Bassam, B.J.; Caetanoanolles, G.; Gresshoff, P.M. Fast and sensitive silver staining of DNA in polyacrylamide gels. Anal. Biochem. 1991, 196, 80-83. [CrossRef]

35. Felsenstein, J. Confidence-limits on phylogenies-An approach using the bootstrap. Evolution 1985, 39, 783-791. [CrossRef] [PubMed]

36. Tamura, K.; Nei, M.; Kumar, S. Prospects for inferring very large phylogenies by using the neighbor-joining method. Proc. Natl. Acad. Sci. USA 2004, 101, 11030-11035. [CrossRef] [PubMed]

37. Kumar, S.; Stecher, G.; Tamura, K. Mega7: Molecular evolutionary genetics analysis version 7.0 for bigger datasets. Mol. Biol. Evol. 2016, 33, 1870-1874. [CrossRef] [PubMed]

38. Liu, K.; Muse, S.V. Powermarker: An integrated analysis environment for genetic marker analysis. Bioinformatics 2005, 21, 2128-2129. [CrossRef] [PubMed]

39. Powell, W.; Morgante, M.; Andre, C.; Hanafey, M.; Vogel, J.; Tingey, S.; Rafalski, A. The comparison of RFLP, RAPD, AFLP and SSR (microsatellite) markers for germplasm analysis. Mol. Breed. 1996, 2, 225-238. [CrossRef]

Sample Availability: Genome survey sequencing data of Luffa cylindrica L. are available from the authors.

(C) 2017 by the authors. Licensee MDPI, Basel, Switzerland. This article is an open access article distributed under the terms and conditions of the Creative Commons Attribution (CC BY) license (http://creativecommons.org/licenses/by/4.0/). 